\title{
Distribución y abundancia de tanatocenosis de foraminíferos bentónicos submareales en el delta del río Colorado
}

\author{
Miguel Ángel Santa Rosa-del Río ${ }^{1, *}$, Guillermo Eliezer Ávila Serrano, \\ Miguel Agustín Téllez-Duarte ${ }^{1}$, Oscar Gonzalez-Yajimovich ${ }^{1}$, Luis Antonio Cupul Magaña ${ }^{1}$
}

${ }^{1}$ Área de Geología, Facultad de Ciencias Marinas, Universidad Autónoma de Baja California, Carretera Tijuana-Ensenada km 103, Apartado postal 453, C.P. 22800 Ensenada, Baja California, México

*miguel_santarosa@hotmail.com

\section{Resumen}

Los foraminíferos bentónicos son indicadores importantes de las condiciones ambientales costeras y marinas por ser muy sensibles a los cambios que ocurren en su entorno, los cuales se reflejan con la presencia y abundancia de especies en las comunidades de tanatocenosis. El objetivo de este trabajo es comparar los cambios espaciales y temporales en la composición y estructura de las comunidades de tanatocenosis de foraminíferos bentónicos en el Alto Golfo de California. Se realizaron cuatro muestreos estacionales (verano, otoño e invierno del 2009, y primavera del 2010) de sedimento en cuatro transectos perpendiculares a la línea de costa (56 muestras), en la zona submareal adyacente a Baja California, entre Campo Don Abel y la parte sur de Isla Montague. Para diferenciar la biocenosis de la tanatocenosis, las muestras fueron teñidas con rosa de bengala. Se trataron en laboratorio por tamizado en húmedo con el fin de eliminar limo y arcilla; fue retenida la fracción entre 63 y $180 \mu \mathrm{m}$ para su análisis y se separaron 300 individuos por muestra. Se identificaron taxonómicamente y se calculó la abundancia relativa para determinar las especies dominantes. Los datos fueron procesados mediante un análisis de agrupamiento en modo Q y R, además se calculó el índice de diversidad de Shannon-Weaver y la dominancia por el índice de Simpson. Por último se realizó un mapa de distribución. Los géneros más abundantes fueron: $A m-$ monia, Cribroelphidium, y Rosalina; éstos representan preferentemente condiciones marinas, lo que muestra cambio en el Alto Golfo de California. También aparecieron en todas las estaciones de muestreo cuatro géneros de foraminíferos aglutinados: Ammoscalaria, Reophax, Anomaloides y Nouria, pero su distribución se centra principalmente en las estaciones más al sur del área de estudio, en los límites del antiguo estuario al norte de San Felipe.

Palabras clave: delta del río Colorado, variaciones espacio-temporales de comunidades de foraminíferos, variación ambiental.

\begin{abstract}
Benthonic foraminifera are important indicators of environmental conditions in coastal and marine areas since they are very sensitive to changes in their surroundings, resulting in presence and abundance of species of the thanatocoenosis communities. The objective of this work is to compare the spatial and temporal changes in the composition and structure of the benthonic-foraminifera thanatocoenosis communities of the upper Gulf of California. Samples were collected seasonally (summer, fall and winter of 2009, and spring of 2010) in the area between Campo Don Abel and the southern end of Isla Montague. Four transects of the subtidal area were run perpendicular to the Baja California coastline, and a total of 56 sediment samples were taken. To differentiate the biocoenosis from thanatocoenosis, the samples were stained with rose bengal. Silt and clay were eliminated by wet sieving and the 63 to 180 um fractions retained for picking. From each sample, 300 individuals were separated, identified and their relative abundance computed to determine the dominant species. Cluster analysis in $Q \& R$ modes was performed on the data and the Shannon-Weaver diversity index calculated; dominance was obtained using Simpson's index, and a distribution map was constructed. The most abundant genera
\end{abstract}


were: Ammonia, Cribroelphidium and Rosalina; these represent preferably marine conditions, which show change in the upper Gulf of California. Also present were four genera of agglutinated foraminifera: Ammoscalaria, Reophax, Anomaloides and Nouria, but their distributions were centered at the southernmost stations of the study area, at the limits of the ancient estuary north of San Felipe.

Keywords: Colorado River Delta, spatial and temporal change in foraminiferal communities, environmental change.

\section{Introducción}

Los foraminíferos bentónicos son una forma de analizar los cambios en la ecología bentónica, ya que se encuentran distribuidos en casi la tercera parte del fondo oceánico, así como en la zona adyacente a la costa. Son organismos unicelulares que existen desde el Cámbrico y que preferentemente se localizan en el medio marino aunque algunas formas ocurren en aguas salobres (Bignot, 1988).

El Alto Golfo, donde se localiza el delta del río Colorado, es un excelente sitio para investigar el impacto antropogénico sobre las comunidades de foraminíferos y otros organismos, debido a que es considerado como un laboratorio natural donde han ocurrido cambios ambientales dramáticos en los últimos 100 años por la desaparición del antiguo estuario (Huerta-Tamayo, 2007).

La cuenca de drenaje del río Colorado comprende un área aproximada de $630000 \mathrm{~km}^{2}$, recorre aproximadamente $1600 \mathrm{~km}$ desde su origen en el estado de Colorado, EE.UU., hasta su desembocadura en el Golfo de California (Thompson, 1968), donde las aguas se enriquecían con material en suspensión rico en nutrientes, dando lugar a una alta productividad bentónica, como lo evidencian los millones de conchas que originan las cordilleras o cheniers localizados a lo largo de la costa de Baja California. Estos depósitos son consecuencia de fuertes corrientes de marea y del oleaje que han ocasionado la concentración de partículas bioclásticas depositadas como grandes acumulaciones de conchas de moluscos (Thompson, 1968; Kowalewsky et al., 1994, 2000).

Con la construcción de las presas Hoover y Glenn Canyon, se controlaron definitivamente las aguas del río Colorado, lo que actualmente ocasiona que el delta se encuentre en un proceso de erosión (van Andel, 1964; Baba et al., 1991; Carriquiry y Sánchez, 1999). Aun cuando ésta se explica por causas antropogénicas, también han ocurrido procesos erosivos naturales por las fluctuaciones del río que ocasionaron la desviación de sus descargas de agua y aporte de sedimentos hacia cuencas adyacentes como el mar de Salton (Thompson, 1968). El impacto ecológico más severo fue por consecuencia del cambio en la hidrología de la zona deltaica, que propició que se transformara de un sistema estuarino a uno antiestuarino (Carbajal et al., 1997; Lavín et al., 1998; Avila-Serrano et al., 2006).

Es importante el entender los procesos erosivos que ocurren actualmente en el delta, para lo cual existen herramientas como el análisis del promediado temporal, el cual se basa principalmente en el estudio de diversas generaciones a través del tiempo, las cuales se ven expuestas a los procesos erosivos ocasionados por la hidrodinámica de la zona. Walker y Bambach (1971) definen el concepto de promediado temporal como: "La acumulación de comunidades vivas locales durante un tiempo requerido para depositar el contenido en el sedimento". Por lo anteriormente comentado y debido a que no se tiene información de las comunidades de foraminíferos bentónicos en la zona submareal antes de las modificaciones al cauce del río Colorado, es importante generar nueva información que sirva como un marco de referencia comparativo para la utilización de estos datos con fines de entender lo que sucede actualmente en el área deltaica.

\section{Objetivo}

Comparar los cambios espaciales y temporales en la estructura de las comunidades de tanatocenosis de foraminíferos bentónicos submareales en el delta del río Colorado, con el fin de determinar si existe un promediado temporal activo de las mismas.

\section{Materiales y métodos}

El delta del río Colorado se localiza entre los $31^{\circ}$ $15^{\prime}$ y $31^{\circ} 50^{\prime}$ de latitud norte y los $114^{\circ} 39^{\prime}$ y $115^{\circ} 05^{\prime}$ de longitud oeste, centrándose este estudio en la zona submareal adyacente a la costa de Baja California (Figura 1), donde en la actualidad ocurre ocasionalmente el único aporte significativo de agua dulce proveniente del propio río durante flujos extraordinarios (Lavín y Sánchez, 1998).

Se realizaron cuatro transectos perpendiculares a la línea de costa con un total de 16 estaciones. Cada una se georeferenció con un sistema de posicionamiento global (GPS por sus siglas en inglés) marca Garmin e-Trex. Para el muestreo de sedimentos superficiales se utilizó una draga tipo "Petite Ponar" con capacidad de $2.4 \mathrm{dm}^{3}$, la cual se lanzó de una embarcación (lancha de pesca de 22 pies). De la draga se obtuvieron submuestras mediante una pala de campo con la que se colectaron $100 \mathrm{~g}$ de sedimento aproximadamente, los cuales se vaciaron en una bolsa de plástico y fijaron con alcohol al $60 \%$ para su procesamiento posterior en laboratorio.

En el laboratorio las muestras se tiñeron con rosa de 


\section{Área de Estudio}

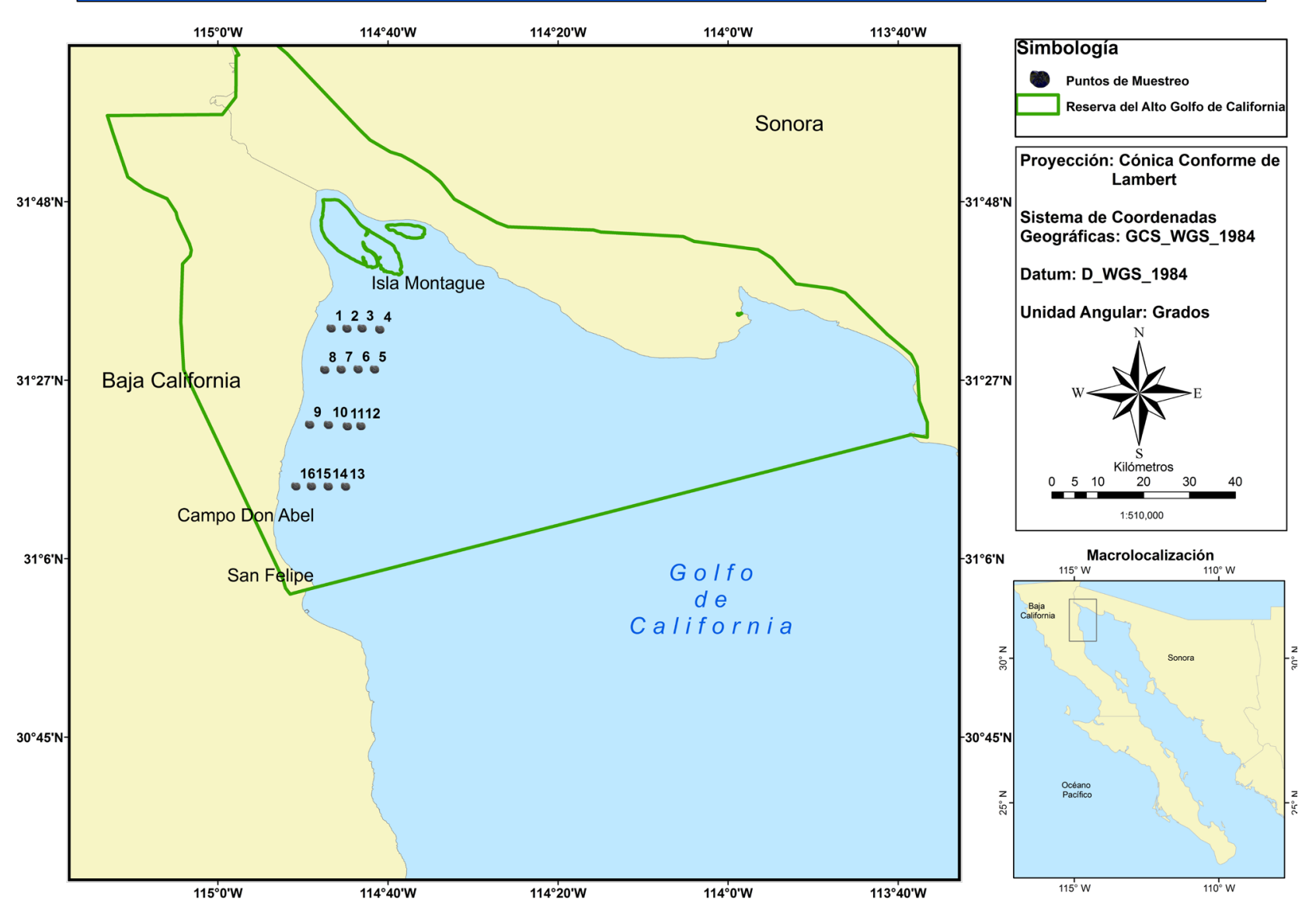

Figura 1. Localización del área de estudio; se indican los transectos con las estaciones de muestreo.

bengala, siguiendo el método descrito por Walton (1952) para diferenciar las biocenosis de las tanatocenosis. Después de un periodo de 48 horas se tamizó en húmedo con una luz de malla de $62 \mu \mathrm{m}$ para eliminar limo y arcilla. Las testas de foraminíferos se concentraron por filtración a través de un papel Whatman número 4 con diámetro de $11 \mathrm{~cm}$, y se secaron en un horno a temperatura controlada de $40^{\circ} \mathrm{C}$. Posteriormente se tamizó la muestra y se separaron las fracciones entre 63 y $180 \mu \mathrm{m}$, por ser donde se concentran mayormente las testas de foraminíferos. Finalmente, se separaron 300 individuos por muestra para su identificación considerando que Lipps et al. (1979) mencionan que éste es el tamaño mínimo de muestra para obtener datos cuantitativos confiables en estudios de diversidad.

Una vez identificados y contabilizados los foraminíferos, con los datos obtenidos se construyó una matriz de 40 casos y 16 variables y fueron procesados mediante un análisis multivariado de agrupamiento en modo "Q" y "R" para comparar las variaciones temporales de la tanatocenosis representados en dendrogramas basados en distancias euclidianas con encadenamiento completo, que cuando son pequeñas indican un alto grado de asociación entre cada una de las variables (Bakus, 2007). Dichos dendrogramas permiten valorar cuál o cuáles son los transectos con mayor similitud con base en el número de individuos identificados por muestra.

El análisis de agrupamiento modo "Q" indica la variabilidad estacional, es decir cómo cambia la distribución de la tanatocenosis de los foraminíferos a lo largo y ancho de la zona de estudio. El modo "R", por otra parte, indica la variabilidad temporal durante las cuatro estaciones del año (2009-2010).

Se hicieron los análisis necesarios para determinar los diferentes índices ecológicos con los programas Statistica versión 7, Excel, Primer versión 5. Estos índices son:

Diversidad de Shannon-Weaver $\left(H^{\prime}\right)$.

Definido como:

$$
H^{\prime}=-\sum_{i=1}^{S} p_{i} \log _{2} p_{i}
$$

Ecuación 1 
Donde $S$ es el número de especies por muestra y $p_{i}$ es la proporción de organismos de la especie respecto al total de organismos (abundancia relativa). Este índice estima la biodiversidad de una población con respecto al número de individuos de cada especie que la conforma.

La equitatividad de Pielou $\left(J^{\prime}\right)$, definida como:

$$
J=H^{\prime} / H^{\prime} \max \quad \text { Ecuación } 2
$$

Donde $H^{\prime}$ corresponde al valor de diversidad esperado y $H^{\prime} \max$ al valor de diversidad máximo. Este índice describe qué tan uniforme es el sistema con base en el modelo hipotético, donde todos los taxones tienen las mismas posibilidades de abundancia. El valor alto indica uniformidad, mientras que el bajo indica, de manera indirecta, que existe dominancia por parte de una(s) especie(s).

También se calculó el índice de dominancia de Simpson $\left(1-\lambda^{\prime}=D\right)$, que se define como:

$$
D=\frac{\sum_{i=1}^{S} n_{i}\left(n_{i}-1\right)}{N(N-1)}
$$

Ecuación 3

Donde $S$ es el número de especies, $N$ es el total de organismos presentes y $n$ es el número de ejemplares por especie. Éste índice indica cuál o cuáles son las especies dominantes en la población, es decir, cuál es la que está conformada con el mayor número de individuos.

Todas las especies identificadas se documentaron por medio de un microscopio electrónico de barrido (Anexo I) marca JEOL, modelo JSM-35C, propiedad de la División de Ciencias de la Tierra del Centro de Investigación Científica y de Educación Superior de Ensenada (CICESE). Asimismo, fueron depositadas en la bodega de paleontología de la Facultad de Ciencias Marinas de la UABC.

\section{Resultados y discusión}

El muestreo de verano no se pudo completar por problemas logísticos, por lo que solo se muestrearon dos transectos (II y III), correspondientes a la parte central del área de estudio.

\subsection{Composición de especies}

En las 56 muestras analizadas se identificaron 40 especies, de las cuales 32 fueron a nivel especie y ocho a nivel de género. La fauna se caracterizó por una baja diversidad: $95 \%$ del total de individuos lo constituyeron 15 especies (Tabla 1), de las cuales tres, Ammonia beccarii parkinsoniana, Rosalina sp1 y Cribroelphidium excavatum, comprendieron el $58 \%$ del total de especies (Figura 2). A. beccarii parkinsoniana fue la especie más abundante, está presente en 55 de las 56 muestras analizadas; es una especie euritópica y euritérmica, y principalmente abunda en ambientes hipersalinos, lo cual concuerda con el actual estado antiestuarino del delta del río Colorado (Walton y Sloan, 1990; Murray, 1991; Debenay et al., 1998; AvilaSerrano et al., 2006). La segunda especie más abundante fue $C$. excavatum. Esta especie es común en ambientes estuarinos (Murray, 1991), por lo que dada su abundancia muy probablemente sean poblaciones acumuladas durante el predominio de las condiciones estuarinas. Rosalina sp1 fue la tercer especie dominante. Se distribuye primordialmente en océano abierto, aunque también llega a zonas lagunares pero con menos frecuencia (Phleger, 1960). Por lo anterior, su abundancia se explica por las condiciones actuales de la parte sur del delta, a diferencia de las condiciones hipersalinas de la parte norte.

Los géneros más diversos en todo el ciclo fueron Cribroelphidium y Quinqueloculina. Del primero de ellos, cinco de las especies conforman el $95 \%$ del total de individuos; y del segundo (Quinqueloculina) tres especies conformaron el $15 \%$ de las más abundantes (Tabla 1). La presencia de este último género sugiere condiciones hipersalinas, de acuerdo a Murray (1973), quien asocia la presencia de miliólidos con ambientes de alta salinidad como actualmente ocurre en el Alto Golfo. En el particular caso de Cribroelphidium, éste se caracteriza por habitar en ambientes lagunares con pastos marinos (Murray, 1991). La ocurrencia conjunta de ambos géneros se puede explicar como resultado del transporte y promediado temporal: por un lado, las amplias praderas del pasto marino Distichilis palmeri en la zona costera y cercanas a la isla Montague constituyen el hábitat ideal de Cribroelphidium sp. bajo las condiciones actuales del delta; y por otro, la presencia de estos organismos hasta la parte sur de la zona de muestreo, donde actualmente no existen condiciones hipersalinas, coincide con la extensión del antiguo estuario del delta del río Colorado (Lavín y Sánchez, 1998; Huerta-Tamayo, 2007).

El género Ammonia se caracterizó por ser el de mayor número de individuos tanto en cada estación del año como en cada transecto muestreado, además de estar dentro de las especies más abundantes del ciclo (Tabla 1). Esta especie se caracteriza por tener una amplia distribución geográfica, así como vivir preferentemente en aguas someras y ambientes con salinidades altas (Murray, 1991; Walton y Sloan, 1990; Debenay et al., 1998). Por tanto es compatible con las condiciones salinas actuales.

Se identificaron cuatro géneros de foraminíferos aglutinados: Ammoscalaria, Reophax, Anomaloides y Nouria (Tabla 2), representados por cinco especies, de las cuales la más abundante es Ammoscalaria pseudospiralis, con un $53 \%$, por otra parte Reophax dentaliformis sólo con el $5 \%$ de abundancia (Tabla 2). La presencia de foraminíferos aglutinados en el área de estudio indica que el antiguo aporte fluvial de las aguas dulces con un $\mathrm{pH}$ más ácido inhibía la calcificación, por lo que los mejor 
Tabla 1. Abundancias total y relativa de foraminíferos bentónicos identificados en el área de estudio durante el ciclo 2009-2010.

\begin{tabular}{|c|c|c|}
\hline Especie & $\begin{array}{l}\text { Abundancia total } \\
\text { (No. de Individuos) }\end{array}$ & $\begin{array}{c}\text { Abundancia relativa } \\
(\%)\end{array}$ \\
\hline Ammonia beccarii parkinsoniana d'Orbigny, 1826 & 5653 & 28.19 \\
\hline Cribroelphidium excavatum Terquem, 1876 & 3206 & 15.99 \\
\hline Rosalina sp. 1 d'Orbigny, 1826 & 2851 & 14.22 \\
\hline Bucella tenerrima Bandy, 1950 & 1297 & 6.47 \\
\hline Cribroelphidium spinatum var. translucens Cushman y Brönnimann, 1948 & 1276 & 6.36 \\
\hline Ammonia sp. Linné, 1758 & 1230 & 6.13 \\
\hline Cribroelphidium gunteri Cushman y Brönnimann, 1948 & 919 & 4.58 \\
\hline Cribroelphidium incertum Cushman y Brönnimann, 1948 & 800 & 3.99 \\
\hline Cribroelphidium poeyanum Cushman y Brönnimann, 1948 & 443 & 2.21 \\
\hline Quinqueloculina sp. 1 d'Orbigny, 1826 & 420 & 2.09 \\
\hline Bulimina marginata d'Orbigny, 1826 & 274 & 1.37 \\
\hline Quinqueloculina sp. 3 d'Orbigny, 1826 & 224 & 1.12 \\
\hline Quinqueloculina sp. 2 d'Orbigny, 1826 & 217 & 1.08 \\
\hline Buliminella elegantissima d'Orbigny, 1826 & 205 & 1.02 \\
\hline Ammoscalaria pseudospiralis Williamson, 1858 & 152 & 0.76 \\
\hline
\end{tabular}

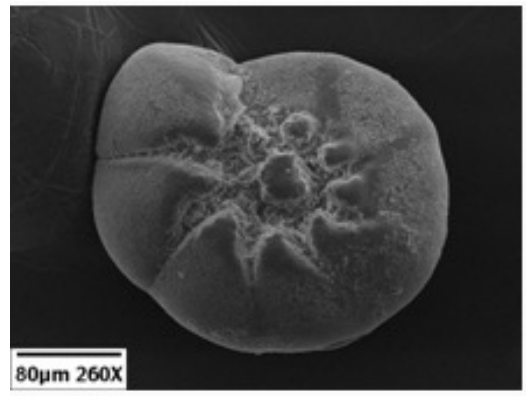

A

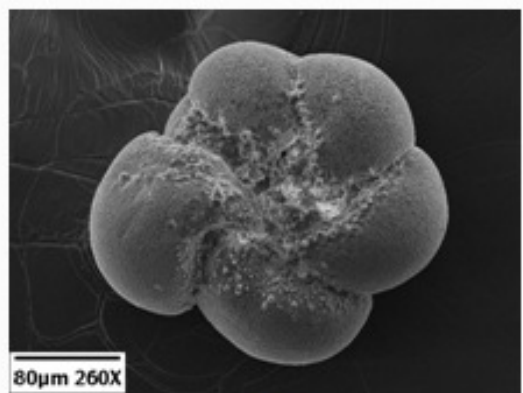

B

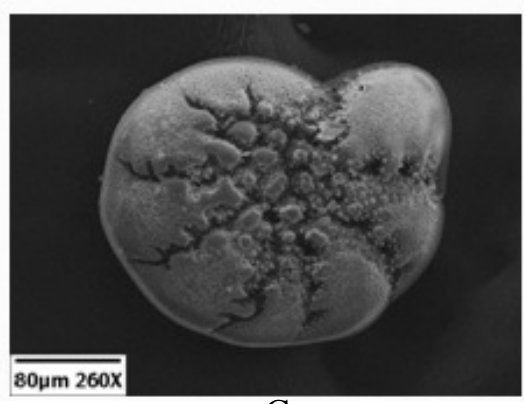

C

Figura 2. Vista ventral de las especies más abundantes en el delta del río Colorado; A) Ammonia beccarii parkinsoniana; B) Cribroelphidium excavatum; C) Rosalina sp1. (Escala a 80 $4 \mathrm{~m}$ a un zoom óptico de $260 \mathrm{X}$ ).

adaptados eran los aglutinados, mientras que las testas calcáreas dominan en aguas más alcalinas (Bernal et al., 2008 en Palafox-Silva, 2011).

\subsection{Distribución y abundancia de especies}

Se representa la asociación de abundancias de foraminíferos en las diferentes temporadas del año en forma de dendrogramas (Figura 3), donde se observa que todos los muestreos se caracterizaron por la presencia de dos grupos dominantes. Sin embargo, las temporadas de verano y primavera responden a un patrón de asociación central y sesgado hacia la costa, mientras que en otoño e invierno la distribución es más homogénea a lo largo y ancho del área estudiada. Esto lo explican seguramente las condiciones climáticas presentes en las temporadas muestreadas, debido a los giros estacionales que se presentan en el Alto Golfo de California, que de acuerdo a lo reportado por Lavín et al.
(1997), a lo largo del año en esta zona se presentan dos tipos de giros, siendo un giro ciclónico durante el verano y un giro anticiclónico durante el invierno. Estos giros aunados a las condiciones físicas que se presentan en el Alto Golfo de California, podrían influenciar en las distribuciones de las comunidades de foraminíferos bentónicos en la zona estudiada.

Con base en las especies más abundantes se realizó un mapa donde se representa la abundancia relativa de cada especie en la zona deltaica (Figura 4). De acuerdo al número de individuos presentes en cada estación, se observa la asociación que hay entre las tres especies dominantes, donde Ammonia está presente en todas las estaciones de muestreo con el mayor número de individuos durante todas las temporadas. Como ya se mencionó, es una especie euritópica, por lo tanto es normal encontrarla en estas latitudes; es también nerítica, que ciertamente corresponde a las profundidades en las que fueron colectadas las muestras 
Tabla 2. Abundancia relativa (expresada en \%) de foraminíferos aglutinados durante el ciclo 2009-2010 en el área de estudio.

\begin{tabular}{|c|c|c|c|c|c|}
\hline Especie & Verano & Otoño & Invierno & Primavera & $\begin{array}{c}\text { Abundancia } \\
\text { relativa } \\
(\%) \\
\end{array}$ \\
\hline Ammoscalaria pseudospiralis Williamson, 1858 & 65 & 50 & 76 & 41 & 53 \\
\hline Nouria sp. Heron-Allen y Earland, 1914 & 7 & 38 & 2 & 36 & 26 \\
\hline Anomalinoides sp. Brotzen, 1942 & 5 & 10 & 16 & 9 & 10 \\
\hline Reophax nanus de Montfort, 1808 & 21 & 0 & 1 & 6 & 6 \\
\hline Reophax dentaliformis de Montfort, 1808 & 2 & 0 & 6 & 8 & 5 \\
\hline
\end{tabular}
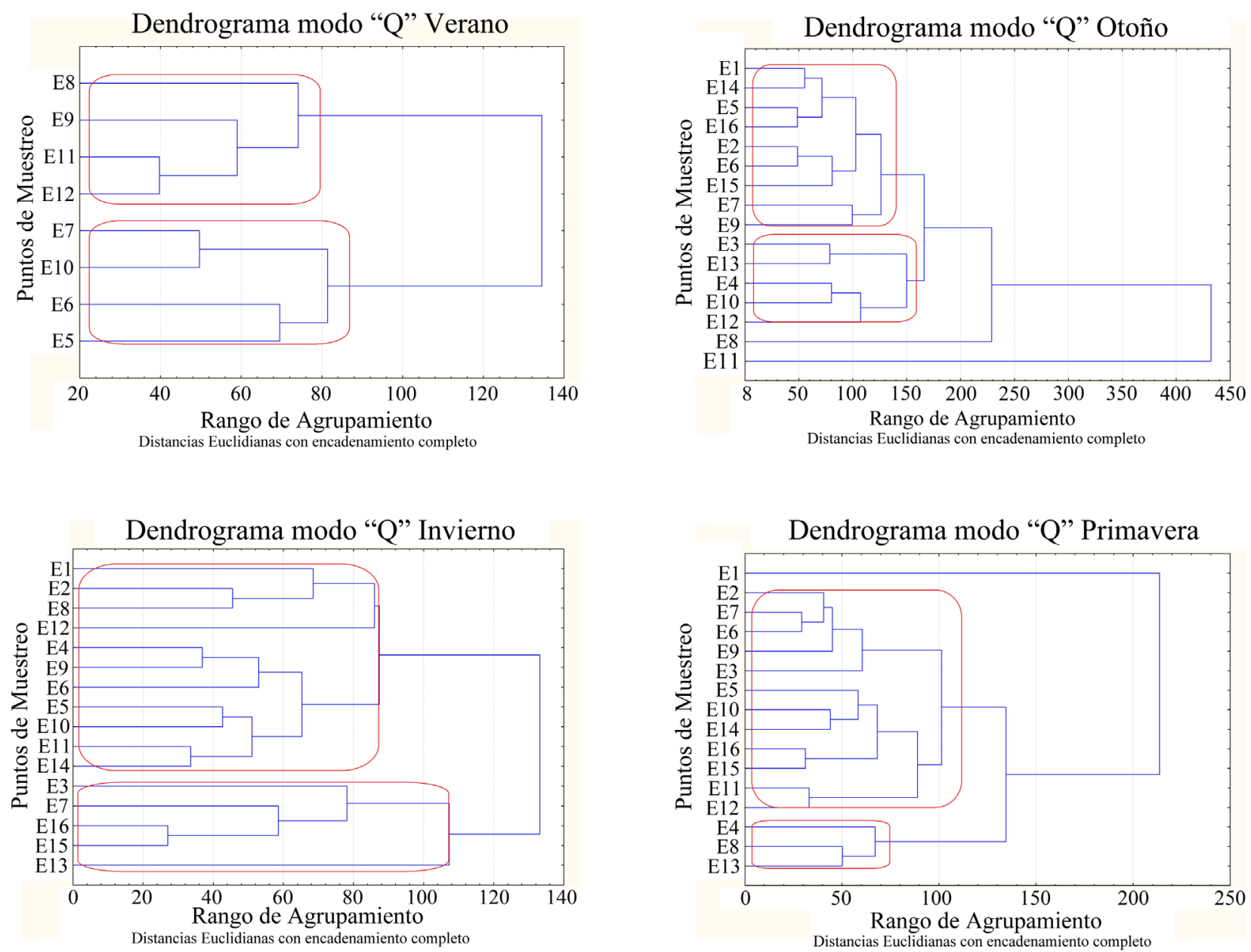

Figura 3. Dendrograma de agrupamiento modo “Q” para el ciclo 2009-2010 (verano, otoño, invierno y primavera).

de sedimento; y por último indica condiciones hipersalinas, que debido al nulo aporte de agua dulce por parte del río Colorado y al alto grado de evaporación que se registra en el Alto Golfo de California, concuerda claramente con la abundancia de este género. Usera y Blázquez (1998) reportan que las condiciones óptimas para el crecimiento de Ammonia se encuentran en un rango de temperatura de entre 24 y $30{ }^{\circ} \mathrm{C}$ y salinidad de $20 \%$ a $40 \%$ o con picos máximos de $50 \%$. Estos datos se correlacionan con los observados en el área deltaica durante los muestreos, ya que se obtuvieron valores promedio de temperatura de 26 ${ }^{\circ} \mathrm{C}$ y salinidades de hasta $48 \%$. Cabe aclarar que cuando la temperatura y salinidad quedan por encima de lo reportado por estos autores, al menos en lo que se refiere a este género, el crecimiento se detiene, pudiendo llegar a la muerte del organismo. 


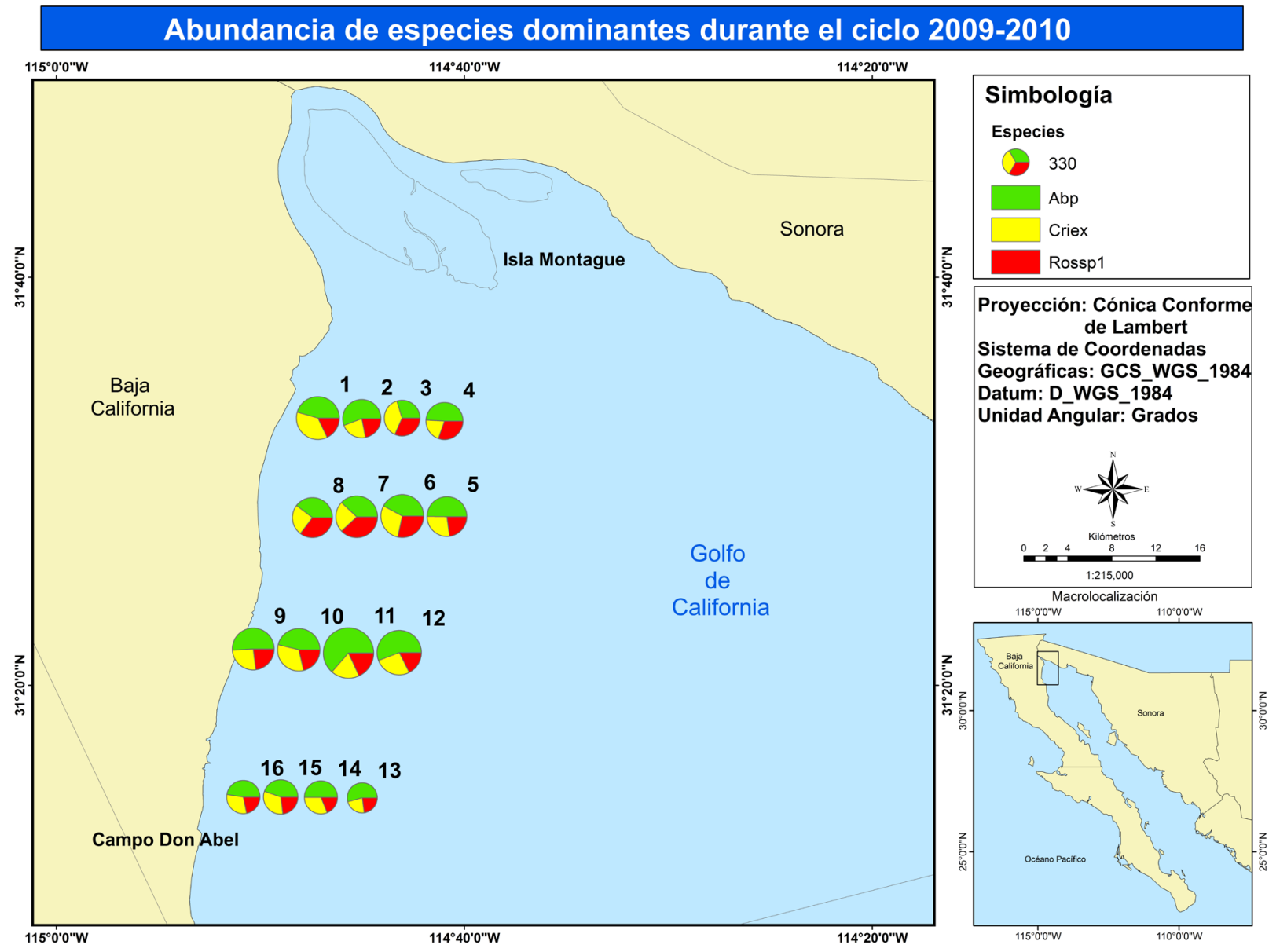

Figura 4. Abundancia relativa de las tres especies dominantes en el delta del río colorado; $(\mathrm{Abp}=$ Ammonia beccarii parkinsoniana, Criex $=$ Cribroelphidium excavatum, Rossp1 = Rosalina sp1). El tamaño de las gráficas es proporcional al número máximo de individuos en cada punto de muestreo.

\subsection{Riqueza, diversidad, equitatividad y dominancia}

La riqueza de especies fue mayor en primavera, con 37 especies, y el verano presentó la menor, con 34 especies, mientras que la mayor abundancia se presentó en el otoño (Tabla 3). Asimismo, las temporadas con mayor similitud en abundancia, fueron en primavera e invierno (Figura 5). Esto probablemente se deba a que el reclutamiento ocurre en esta temporada. Jorissen y Wittling (1999), mencionan que para el Alto Golfo de California en la temporada de otoño se presenta un pulso reproductivo asociado a un afloramiento de fitoplancton, por lo tanto probablemente este aumento en la abundancia podría responder a estos reclutamientos. Como se mencionó anteriormente, el muestreo de verano fue el que menor número de individuos presento debido a problemas de logística.

Los índices estadísticos de diversidad (H'), equitatividad (J') y dominancia (D) se muestran en la Tabla 3. El primero presentó valores durante todo el ciclo entre 3.4 y 3.2 , en primavera y verano están los valores más altos y en invierno fue el más bajo. Esto se relaciona con lo comentado anteriormente, en cuanto a la abundancia de especies en temporadas cálidas y se debe al reclutamiento presentado en dichas temporadas que es ocasionado por los afloramientos de fitoplancton (Jorissen y Wittling, 1999).

Con respecto a la equitatividad, ésta mostró poca variación ( 0.6 y 0.7$)$, donde los valores más altos fueron en verano y otoño, mientras que primavera e invierno son las temporadas con los más bajos. Esto indica que la comunidad se encuentra distribuida de manera homogénea en toda el área de estudio, debido a que la mayor abundancia de individuos se compone de un número muy reducido de especies.

Para el caso de la dominancia (D) se puede ver que pocas especies son dominantes. En primavera y verano fue la mayor (0.9), mientras que otoño e invierno tienen los valores más bajos (0.8). Sobresale Ammonia beccarii parkinsoniana como la especie más dominante que es cosmopolita, euritérmica, euritópica y eurihalina y, a pesar 
Tabla 3. Índices de diversidad, equitatividad y dominancia para cada estación del año y para el ciclo completo de muestreos en el área deltaica.

\begin{tabular}{lccccc}
\hline \multicolumn{1}{c}{ Periodo } & Riqueza & $\begin{array}{c}\text { Número total } \\
\text { de individuos }\end{array}$ & $\begin{array}{c}\text { Equitatividad de Pielou } \\
(\mathbf{N})\end{array}$ & $\begin{array}{c}\text { Índice de diversidad } \\
\text { Shannon-Weaver } \\
\text { H'(Log2) }\end{array}$ & $\begin{array}{c}\text { Índice de dominancia } \\
\text { de Simpson }\end{array}$ \\
\hline Primavera & $(\mathbf{S})$ & 5089 & 0.6 & 3.4 & 0.9 \\
Verano & 37 & 2530 & 0.7 & 3.4 & 0.9 \\
Otoño & 34 & 6393 & 0.7 & 3.3 & 0.8 \\
Invierno & 35 & 4991 & 0.6 & 3.2 & 0.8 \\
Ciclo 2009-2010 & 35 & 19003 & 0.6 & 3.4 & 0.9 \\
\hline
\end{tabular}

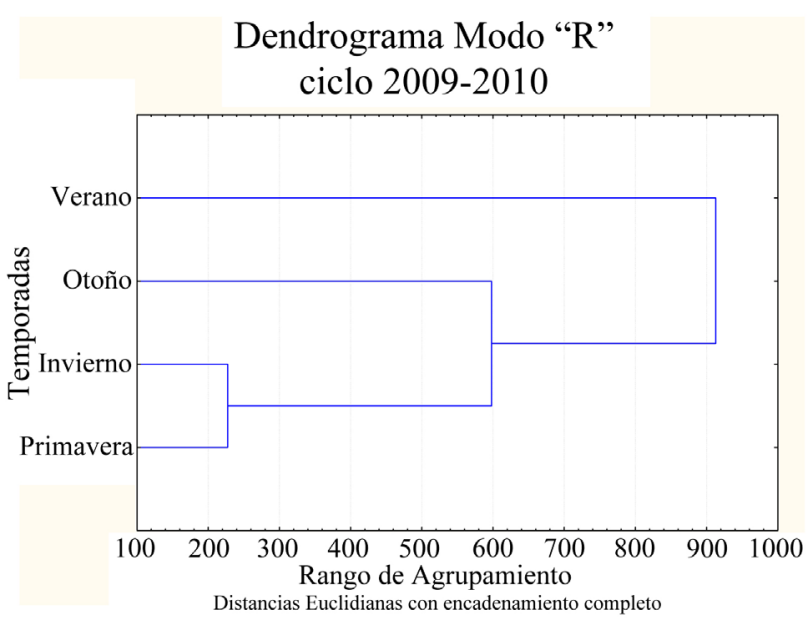

Figura 5. Dendrograma de similitud modo "R" de la abundancia de foraminíferos bentónicos por estaciones del año.

de ser principalmente de ambiente oceánico (mar abierto), también se encuentra en ambientes estuarinos. Usera y Blázquez (1998), reportan que dicha especie responde a dos estrategias de reproducción: 1) "k", es cuando se encuentra en ambientes estables como el oceánico; y 2) "r", es cuando se encuentra en zonas inestables como lagunares y estuarinas. En nuestro estudio se observa el aumento de la abundancia y dominancia de Ammonia beccarii parkinsoniana, lo cual implica un comportamiento de estrategia " $r$ ", al ser organismos de tamaño pequeño y al presentar un aumento de la densidad de individuos. Respecto a sus formas, se presentaron con mayor abundancia las macrosféricas, en comparación con las microsféricas. El aumento de dichas formas puede estar relacionado con un ambiente próximo al óptimo para el crecimiento y desarrollo de esta especie (Usera y Blázquez, 1998).

\section{Conclusiones}

- La especie más abundante fue Ammonia beccarii parkinsoniana; y el aglutinado más abundante Ammoscalaria pseudospiralis, con la mayor ocurrencia en invierno.

- Los géneros más diversos fueron Cribroelphidium y
Quinqueloculina, con 6 especies cada uno.

- Existe poca equitatividad en el área de estudio debido a que la mayor abundancia de individuos se compone de un número muy reducido de especies.

- De acuerdo con el índice de dominancia de Simpson, las tres especies dominantes fueron: Ammonia beccarii parkinsoniana, Cribroelphidium excavatum y Rosalina sp1.

- La mayor abundancia de organismos se presentó en el otoño, mientras que primavera e invierno fueron las temporadas con mayor similitud en cuanto al número de individuos colectados.

- La primavera y el verano presentaron un patrón de distribución central y sesgado hacia la costa, pero en otoño e invierno su distribución fue más uniforme a lo largo y ancho del área de estudio.

\section{Agradecimientos}

Este trabajo fue apoyado por la Universidad Autónoma de Baja California, Facultad de Ciencias Marinas programa 360 y por el CONACyT, convenio CB-2007-01-84710. En especial queremos agradecer al Dr. Robert G. Douglas, profesor emérito de la Universidad del Sur de California (U.S.C.), por su colaboración en la identificación de los foraminíferos, y al Tec. Luis Carlos Gradilla Martínez del laboratorio del microscopio electrónico de barrido, propiedad del departamento de Ciencias de la Tierra del CICESE, por su ayuda con las imágenes electrónicas.

\section{Referencias}

Avila-Serrano, G.E, Flessa, K.W., Téllez-Duarte, M.A., Cintra-Buenrostro, C.E., 2006, Distribución de la macrofauna intermareal del Delta del Río Colorado, norte del Golfo de California, México: Ciencias Marinas, 32, 649-661.

Baba, J., Peterson, C.D., Schrader, H.J., 1991, Modern fine-grained sediment in the Gulf of California during the last century, en Dauphin, J.P., Simoneit, B.R.T. (eds.), The Gulf and Peninsular Province of the Californias: Tulsa, Oklahoma, EUA, American Association of Petroleum Geologists, 569-587.

Bakus, G.J., 2007, Quantitative Analysis of Marine Biological Communities: Field Biology and Environment: Hoboken, New Jersey, Wiley-Interscience, $435 \mathrm{p}$. 
Bernal, G., Ruiz-Ochoa, M.A., Piedrahita, M.T., Restrepo, E., 2008, Foraminíferos en los sedimentos superficiales del sistema lagunar de Cispatá y la interacción río Sinú-Mar Caribe colombiano: Boletín de Ciencias de la Tierra, 23, 5-20.

Bignot, G., 1988, Los microfósiles - Los diferentes grupos, aplicaciones paleobiológicas y geológicas: Madrid, Editora Paraninfo, 284 p.

Carbajal, N., Sousa, A., Durazo, R., 1997, A numerical model of the exROFI of the Colorado River: Journal of Marine Systems, 12, 17-33.

Carriquiry, J.D., Sánchez, A., 1999, Sedimentation in the Colorado River delta and Upper Gulf of California after nearly a century of discharge loss: Marine Geology, 158, 125-145.

Debenay, J.P., Bénéteau, E., Zhang, J., Stouff, V., Geslin, E., Redois, F., Fernandez-Gonzalez, M., 1998, Ammonia becarii and Ammonia tepida (Foraminifera): morphofunctional arguments for their distinction: Marine Micropaleontology, 34, 235-244 .

Huerta-Tamayo, L., 2007, Biocenosis y tanatocenosis de foraminíferos bentónicos como indicadores de cambios ambientales en el delta del río Colorado: Ensenada, Baja California, Universidad Autónoma de Baja California, tesis de maestría, $76 \mathrm{p}$.

Jorissen, F.J., Wittling, I., 1999, Ecological evidence from live-dead comparisons of benthic foraminiferal faunas off Cape Blanc (Northwest Africa): Palaeogeography, Palaeoclimatology, Palaeoecology, 149, 151-170.

Kowalewsky, M., Flessa, K.W., Aggen, J.A., 1994, Taphofacies analysis of Recent shelly cheniers (beach ridges) northeastern Baja California: Facies, 31, 209-241.

Kowalewsky, M., Avila Serrano, G.E., Flessa, K.W., Goodfriend, G.A., 2000, Dead delta's former productivity: Two trillion shells at the mouth of the Colorado River: Geology, 28, 1059-1062.

Lavín, M.F., Sánchez, S., 1998, On how the Colorado River affected the hydrography of the Upper Gulf of California: Continental Shelf Research, 19, 1545-1560.

Lavín, M.F., Beier, E., Badán, A., 1997, Estructura hidrográfica y circulación del Golfo de California: Escalas estacional e interanual, en Lavín, M.F. (ed.), Contribuciones a la Oceanografía Física en México: Ciudad de México, Unión Geofísica Mexicana, 41-171.

Lavín, M.F., Godínez, V.M., Álvarez, L.G., 1998, Inverse-estuarine features of the Upper Gulf of California: Estuarine, Coastal and Shelf Science, 47, 769-795.
Lipps, J.H., Berger, W.H., Buzas, M.A., Douglas, R.G., Ross, C.A., 1979, Foraminiferal Ecology and Paleoecology: Houston, Texas, Society of Economic Paleontologists \& Mineralogists, 198 p.

Murray, J.W. 1973, Distribution and Ecology of Living Benthic Foraminiferids: Nueva York, Crane Russak and Company, 274 p.

Murray, J.W., 1991, Ecology and Paleoecology of Benthic Foraminifera: Essex, Reino Unido, Longman Scientific and Technical, 397 p.

Palafox-Silva, L., 2011, Composición y estructura de las tanatocenosis de foraminíferos bentónicos submareales en el delta del Colorado: Ensenada, Baja California, Universidad Autónoma de Baja California, tesis de licenciatura, $35 \mathrm{p}$.

Phleger, F.B., 1960, Ecology and Distribution of Recent Foraminifera: Baltimore, EUA, Johns Hopkins Press, 297 p.

Thompson, R.W., 1968, Tidal flat sedimentation on the Colorado River Delta, northwestern Gulf of California: Boulder, Colorado, Geological Society of America, $133 \mathrm{p}$.

Usera, J., Blázquez, A.M., 1998, Interpretación de la Dinámica Poblacional de Ammonia beccarii (Linné, 1758) (Foraminiferida, Protoctista) en el Cuaternario Continental de la Laguna de Villena (Alicante, España): Coloquios de Paleontología, 49, 145-167.

van Andel, T.H., 1964, Recent marine sediments of the Gulf of California, en van Andel, T.J., Shor, G.G. (eds.), Marine Geology of the Gulf of California: A Symposium: Tulsa, Oklahoma, EUA, American Association of Petroleum Geologists, 216-310.

Walker, K.R., Bambach, R.K., 1971, The significance of fossil assemblages from fine-grained sediments: time-averaged communities: Geological Society of America Abstracts with Programs, 3, 783-784.

Walton, W.R., 1952, Techniques for the recognition of living foraminifera: Contributions from the Cushman Foundation for Foraminiferal Research, 3, 56-60.

Walton, W.R., Sloan, B.J., 1990, The genus Ammonia Brünnich, 1772, its geographic distribution and morphologic variability: Journal of Foraminiferal Research, 20, 128-156.

Manuscrito recibido: Febrero 24, 2011.

Manuscrito corregido recibido: Agosto 30, 2011.

Manuscrito aceptado: Septiembre 1, 2011.

\section{Anexo}

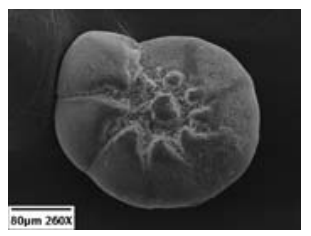

Orden: Foraminiferida Eichwald, 1830 Suborden: Rotaliina Delage y Hérouard, 1896 Familia: Ammoniidae Saidova, 1981 Género: Ammonia Brünnich, 1772 Especie: Ammonia beccarii Linné 1758 Subespecie: Ammonia beccarii parkinsoniana d'Orbigny, 1826

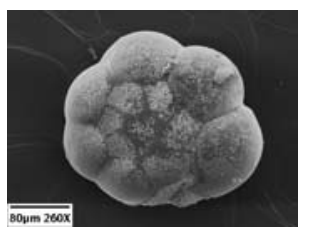

Orden: Foraminiferida Eichwald, 1830

Suborden: Rotaliina Delage y Hérouard, 1896

Familia: Ammoniidae Saidova, 1981

Género: Ammonia Brünnich, 1772

Especie: Ammonia sp.

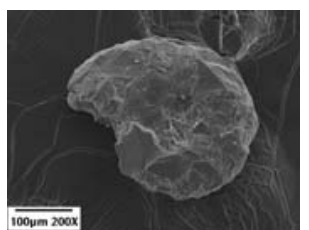

Orden: Foraminiferida Eichwald, 1830

Suborden: Textulariina Delage y Hérouard, 1896

Familia: Discamminidae Mikhalevich, 1980

Género: Ammoscalaria Höglund, 1947

Especie: Ammoscalaria pseudospiralis Williamson 


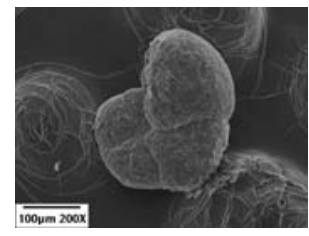

Orden: Foraminiferida Eichwald, 1830

Suborden: Rotaliina Delage y Hérouard, 1896

Familia: Heterolepidae González-Donoso, 1969

Género: Anomalinoides Brotzen, 1942

Especie: Anomalinoides sp.

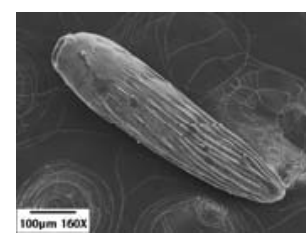

Orden: Foraminiferida Eichwald, 1830

Suborden: Rotaliina Delage y Hérouard, 1896

Familia: Bolivinidae Glaessner, 1937

Género: Bolivina d'Orbigny, 1839

Especie: Bolivina acutula

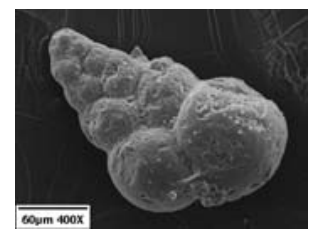

Orden: Foraminiferida Eichwald, 1830

Suborden: Rotaliina Delage y Hérouard, 1896 Familia: Bolivinidae Glaessner, 1937

Género: Bolivina d'Orbigny, 1839

Especie: Bolivina sp. 1

Orden: Foraminiferida Eichwald, 1830

Imagen no disponible

Suborden: Rotaliina Delage y Hérouard, 1896 Familia: Bolivinidae Glaessner, 1937

Género: Bolivina d'Orbigny, 1839

Especie: Bolivina sp. 2

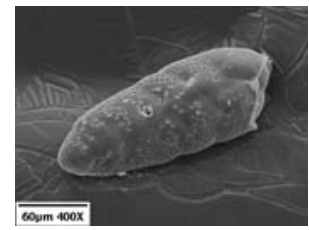

Orden: Foraminiferida Eichwald, 1830

Suborden: Rotaliina Delage y Hérouard, 1896

Familia: Bolivinidae Glaessner, 1937

Género: Bolivina d'Orbigny, 1839

Especie: Bolivina sp. 3

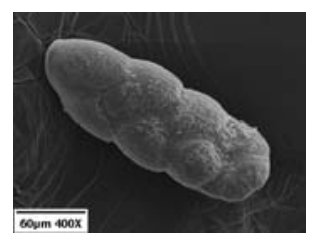

Orden: Foraminiferida Eichwald, 1830

Suborden: Rotaliina Delage y Hérouard, 1896

Familia: Bolivinidae Glaessner, 1937

Género: Bolivina d'Orbigny, 1839

Especie: Bolivina sp. 4

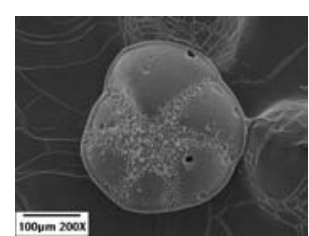

Orden: Foraminiferida Eichwald, 1830

Suborden: Rotaliina Delage y Hérouard, 1896

Familia: Trichohyalidae Saidova, 1981

Género: Bucella Bandy, 1950

Especie: Bucella tenerrima

Orden: Foraminiferida Eichwald, 1830

Imagen no disponible

Familia: Buliminidae Jones, 1875

Género: Bulimina d'Orbigny, 1826

Especie: Bulimina colombiaensis

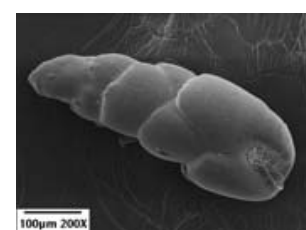

Orden: Foraminiferida Eichwald, 1830

Suborden: Rotaliina Delage y Hérouard, 1896

Familia: Buliminidae Jones, 1875

Género: Bulimina d'Orbigny, 1826

Especie: Bulimina denudata 


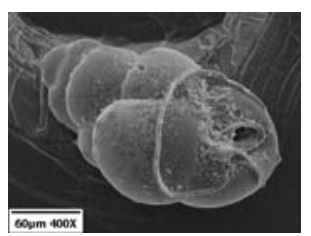

Orden: Foraminiferida Eichwald, 1830

Suborden: Rotaliina Delage y Hérouard, 1896

Familia: Buliminidae Jones, 1875

Género: Bulimina d'Orbigny, 1826

Especie: Bulimina marginata d'Orbigny, 1826

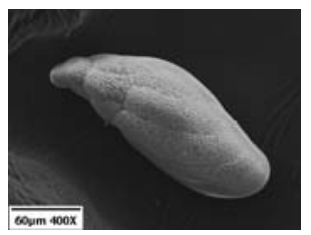

Orden: Foraminiferida Eichwald, 1830

Suborden: Rotaliina Delage y Hérouard, 1896

Familia: Turrilinidae Cushman, 1927

Género: Buliminella Cushman, 1911

Especie: Buliminella elegantissima d'Orbigny, 1826

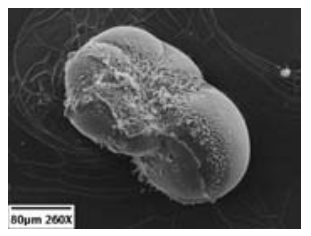

Orden: Foraminiferida Eichwald, 1830

Suborden: Rotaliina Delage y Hérouard, 1896

Familia: Baggininae Cushman, 1927

Género: Cancris de Montfort, 1808

Especie: Cancris sp.

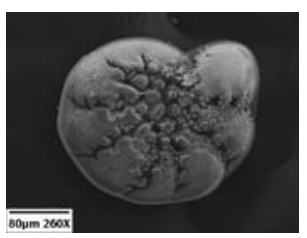

Orden: Foraminiferida Eichwald, 1830

Suborden: Rotaliina Delage y Hérouard, 1896

Familia: Elphidiidae Galloway, 1933

Género: Cribroelphidium Cushman y Brönnimann, 1948

Especie: Cribroelphidium excavatum Terquem, 1876

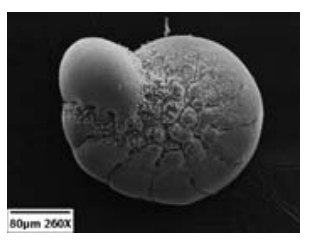

Orden: Foraminiferida Eichwald, 1830

Suborden: Rotaliina Delage y Hérouard, 1896

Familia: Elphidiidae Galloway, 1933

Género: Cribroelphidium Cushman y Brönnimann, 1948

Especie: Cribroelphidium gunteri Cole, 1931

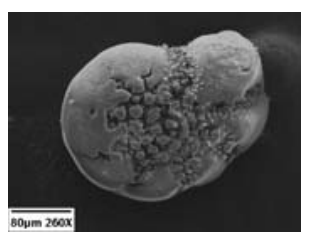

Orden: Foraminiferida Eichwald, 1830

Suborden: Rotaliina Delage y Hérouard, 1896

Familia: Elphidiidae Galloway, 1933

Género: Cribroelphidium Cushman y Brönnimann, 1948

Especie: Cribroelphidium incertum

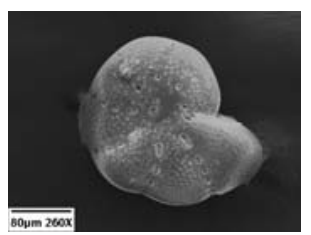

Orden: Foraminiferida Eichwald, 1830

Suborden: Rotaliina Delage y Hérouard, 1896

Familia: Elphidiidae Galloway, 1933

Género: Cribroelphidium Cushman y Brönnimann, 1948

Especie: Cribroelphidium poeyanum

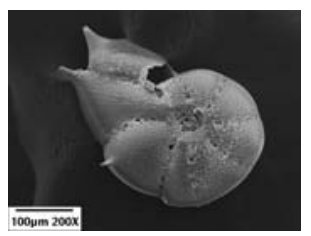

Orden: Foraminiferida Eichwald, 1830

Suborden: Rotaliina Delage y Hérouard, 1896

Familia: Elphidiidae Galloway, 1933

Género: Cribroelphidium Cushman y Brönnimann, 1948

Especie: Cribroelphidium spinatum

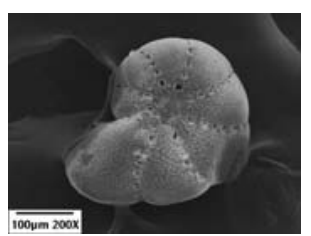

Orden: Foraminiferida Eichwald, 1830

Suborden: Rotaliina Delage y Hérouard, 1896

Familia: Elphidiidae Galloway, 1933

Género: Cribroelphidium Cushman y Brönnimann, 1948

Especie: Cribroelphidium spinatum var. translucens 


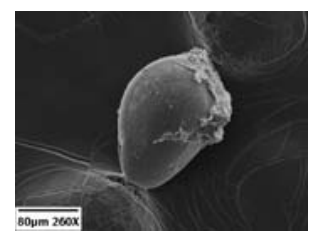

Orden: Foraminiferida Eichwald, 1830

Suborden: Lagenina Delage y Hérouard, 1896

Familia: Ellipsolagenidae Silvestri, 1923

Género: Fissurina Reuss, 1850

Especie: Fissurina sp.

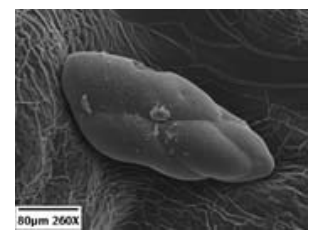

Orden: Foraminiferida Eichwald, 1830

Suborden: Globigerinina Delage y Hérouard, 1896

Familia: Fursenkoinidae Loeblich y Tappan, 1961

Género: Fursenkoina Loeblich y Tappan, 1961

Especie: Fursenkoina pontoni

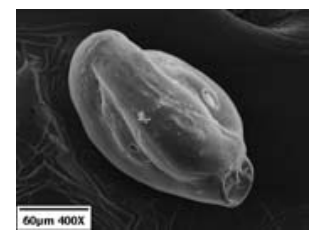

Orden: Foraminiferida Eichwald, 1830

Suborden: Miliolina Delage y Hérouard, 1896

Familia: Hauerinidae Schwager, 1876

Género: Pseudomassilina Lacroix, 1938

Especie: Pseudomassilina sp.

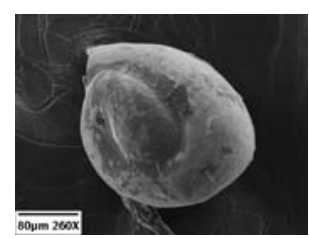

Orden: Foraminiferida Eichwald, 1830

Suborden: Miliolina Delage y Hérouard, 1896

Familia: Hauerinidae Schwager, 1876

Género: Miliolinella Wiesner, 1931

Especie: Miliolinella sp.

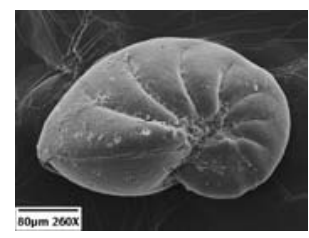

Orden: Foraminiferida Eichwald, 1830

Suborden: Rotaliina Delage y Hérouard, 1896

Familia: Nonionidae Schultze, 1854

Género: Nonionoides Saidova, 1975

Especie: Nonionoides basispinata

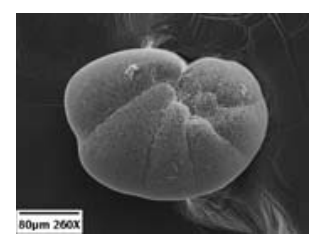

Orden: Foraminiferida Eichwald, 1830

Suborden: Rotaliina Delage y Hérouard, 1896

Familia: Nonionidae Schultze, 1854

Género: Nonionoides Saidova, 1975

Especie: Nonionoides stella

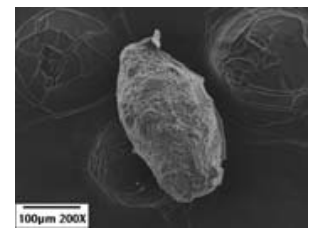

Orden: Foraminiferida Eichwald, 1830

Suborden: Textulariina Delage y Hérouard, 1896

Familia: Ammosphaeroidininae

Género: Nouria Heron-Allen y Earland, 1914

Especie: Nouria sp.

Orden: Foraminiferida Eichwald, 1830

Suborden: Lagenina Delage y Hérouard, 1896

\section{Imagen no disponible}

Familia: Polymorphinidae d'Orbigny, 1839

Subfamilia: Polymorphininae

Género: Globullina d'Orbigny, 1839

Especie: Globullina sp.

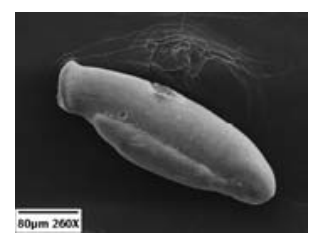

Orden: Foraminiferida Eichwald, 1830

Suborden: Miliolina Delage y Hérouard, 1896

Familia: Hauerinidae Schwager, 1876

Género: Quinqueloculina d'Orbigny, 1826

Especie: Quinqueloculina exculpta 

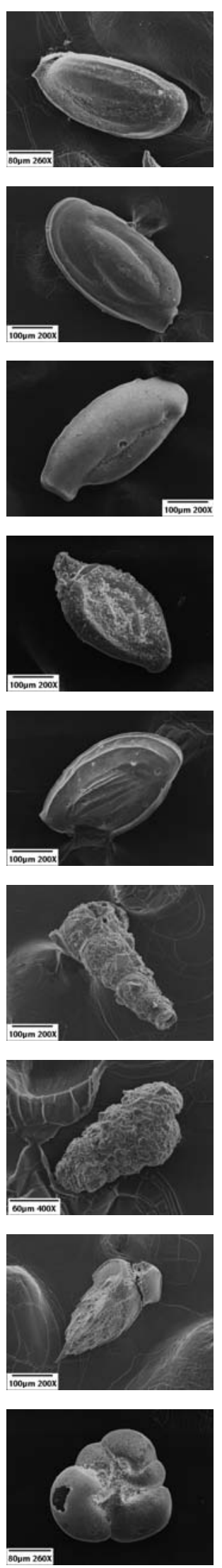

Orden: Foraminiferida Eichwald, 1830

Suborden: Miliolina Delage y Hérouard, 1896

Familia: Hauerinidae Schwager, 1876

Género: Quinqueloculina d'Orbigny, 1826

Especie: Quinqueloculina poeyana d'Orbigny, 1826

Orden: Foraminiferida Eichwald, 1830

Suborden: Miliolina Delage y Hérouard, 1896

Familia: Hauerinidae Schwager, 1876

Género: Quinqueloculina d'Orbigny, 1826

Especie: Quinqueloculina sp. 1

Orden: Foraminiferida Eichwald, 1830

Suborden: Miliolina Delage y Hérouard, 1896

Familia: Hauerinidae Schwager, 1876

Género: Quinqueloculina d'Orbigny, 1826

Especie: Quinqueloculina sp. 2

Orden: Foraminiferida Eichwald, 1830

Suborden: Miliolina Delage y Hérouard, 1896

Familia: Hauerinidae Schwager, 1876

Género: Quinqueloculina d'Orbigny, 1826

Especie: Quinqueloculina sp. 3

Orden: Foraminiferida Eichwald, 1830

Suborden: Miliolina Delage y Hérouard, 1896

Familia: Hauerinidae Schwager, 1876

Género: Quinqueloculina d'Orbigny, 1826

Especie: Quinqueloculina sp. 4

Orden: Foraminiferida Eichwald, 1830

Suborden: Textulariina Delage y Hérouard, 1896

Familia: Hormosinidae Haeckel, 1894

Género: Reophax de Montfort, 1808

Especie: Reophax dentaliformis

Orden: Foraminiferida Eichwald, 1830

Suborden: Textulariina Delage y Hérouard, 1896 Familia: Hormosinidae Haeckel, 1894

Género: Reophax de Montfort, 1808

Especie: Reophax nanus

Orden: Foraminiferida Eichwald, 1830

Suborden: Rotaliina Delage y Hérouard, 1896

Familia: Reussellidae Cushman, 1933

Género: Reusella Galloway, 1933

Especie: Reusella sp.

Orden: Foraminiferida Eichwald, 1830

Suborden: Rotaliina Delage y Hérouard, 1896

Familia: Rosalinidae Reiss, 1963

Género: Rosalina d'Orbigny, 1826

Especie: Rosalina colombiensis 


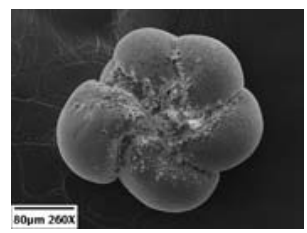

Orden: Foraminiferida Eichwald, 1830

Suborden: Rotaliina Delage y Hérouard, 1896

Familia: Rosalinidae Reiss, 1963

Género: Rosalina d'Orbigny, 1826

Especie: Rosalina sp. 